\title{
Spatio-Temporal Modelling of the p53-mdm2 Oscillatory System
}

\author{
K.E. Gordon ${ }^{1}$, I.M.M. van Leeuwen ${ }^{1,2}$, S. Laín ${ }^{2}$, M.A.J. Chaplain ${ }^{1, *}$ \\ ${ }^{1}$ Department of Mathematics, University of Dundee, WTB/MSI-Complex, \\ Old Hawkhill, DD1 5EH Dundee, Scotland, UK \\ ${ }^{2}$ Department of Surgery and Oncology, University of Dundee, \\ Ninewells Hospital, DD1 9SY Dundee, Scotland, UK
}

\begin{abstract}
In this paper we investigate the role of spatial effects in determining the dynamics of a subclass of signalling pathways characterised by their ability to demonstrate oscillatory behaviour. To this end, we formulate a simple spatial model of the p53 network that accounts for both a negative feedback and a transcriptional delay. We show that the formation of protein density patterns can depend on the shape of the cell, position of the nucleus, and the protein diffusion rates. The temporal changes in the total amounts of protein are also subject to spatial influences. The level of DNA damage required to induce sustained oscillations, for instance, depends on the morphology of the cell. The model also provides a new interpretation of experimentally observed undamped oscillations in p53 levels in single cells. Our simulations reveal that alternate sequences of highand low-amplitude oscillations can occur. We propose that the digital pulses may correspond to snap-shots of our high-amplitude sequences. Shorter waiting-times between subsequent time-lapse fluorescence microscopy images in combination with lower detection thresholds may reveal the irregular high-frequency oscillations suggested by our spatial model.
\end{abstract}

Key words: tumour suppressor, cancer, signalling pathway, limit cycle, pulse.

AMS subject classification: 34C15, 34C23, 35B05, 92C40, 92C50

${ }^{*}$ Corresponding author. E-mail: chaplain@maths.dundee.ac.uk 


\section{Introduction}

During the cell cycle, key proteins undergo cyclic synthesis and degradation, thereby triggering progression through the cell-cycle phases. It is notable that, in recent years, the components of several regulatory networks have been proposed to display similar oscillatory behaviour. Among such networks are the NF- $\kappa \mathrm{B}[15,28]$, Hes1 [4, 12], Wnt $[6,46]$ and p53 [18, 17] signalling pathways. Given the biomedical importance of these pathways, the molecular basis and biological implications of their oscillations have attracted extensive attention from both experimentalists and theoreticians.

Although the signalling pathways above differ in their components and biological outcomes, the oscillations are believed to share a common underlying mechanism: a negative feedback loop (NFL) combined with a transcriptional delay [26, 34, 43]. Whereas much effort has been invested in understanding the temporal dynamics of these systems, spatial insight is still lacking. Recent evidence suggests, however, that cell size and shape can substantially influence intracellular signal transduction [24, 29]. As expressed by [14]: "it has become apparent that distinct spatio-temporal activation profiles of the same repertoire of signalling proteins result in different gene activation patterns and diverse physiological responses." With this in mind, here we formulate a continuum spatial model for the p53 pathway and investigate its behaviour. As the model is based on general principles, our predictions have relevance for other NFL networks.

The p53 protein, also known as the guardian of the genome, is a well-described tumour suppressor that is dysfuntional in nearly half of all human cancers [44]. Its oscillatory response to DNA-damaging stress emerges as a natural consequence of a transcriptional feedback loop, in which p53 promotes the synthesis of its main antagonist, mdm2 [11, 48]. Existing mathematical models have attempted to describe the damped oscillations in p53 and mdm2 levels in cell populations (e.g. [18, 25, 42]), and the undamped pulses observed in individual cells (e.g. [17, 5, 22, 45]), after exposure to ionising radiation. As our aim is to explore the impact of spatial effects rather than to develop a detailed, complex model of the p53-mdm2 pathway, the analysis below is based on a minimal description of the network demonstrating cyclic behaviour. To facilitate introducing our model, we first present a purely kinetic approach (Section 2.), which we then extend to include spatial features (Section 3.). Further technical details and the parameter values used in the simulations can be found in Appendix. In Section 4., we investigate how spatial density patterns and total concentrations evolve under stress conditions for three morphologically different cells. We conclude (Section 5.) with a critical discussion of our model predictions and their biological implications.

\section{The kinetic p53-mdm2 model}

The structure of our network is shown in Fig.1. It accounts for two molecular components, p53 and $\mathrm{mdm} 2$, and the following six phenomena: (1) basal p53 synthesis; (2) mdm2-independent p53 degradation; (3) mdm2-mediated p53 elimination; (4) basal, p53-independent mdm2 synthesis; (5) p53-induced mdm2 synthesis; and (6) mdm2 degradation. According to the reaction scheme 
shown in Fig.1, the changes in the concentrations of p53 and mdm 2 are given by:

$$
\begin{aligned}
& \frac{\mathrm{d}[P]}{\mathrm{d} t}=r_{1}-r_{2}-r_{3}, \\
& \frac{\mathrm{d}[M]}{\mathrm{d} t}=r_{4}+r_{5}-r_{6},
\end{aligned}
$$

where $r_{i}$, for $i=1, \ldots, 6$, is the rate of reaction $i$. For simplicity, we assume that the basal $\mathrm{p} 53$ and mdm 2 synthesis rates, $r_{1}$ and $r_{4}$, remain constant in time and that basal degradation rates, $r_{2}$ and $r_{6}$, are proportional to the corresponding substrate concentrations. Experimental evidence has shown that mdm2 promotes p53 degradation [11] and that this function is inhibited under DNA damage [39]. We have incorporated these observations by making $r_{3}$ an increasing function of mdm 2 as well as a decreasing function of the amount of DNA damage. Finally, reaction 5 represents the negative feedback loop in which p53 transactivates expression of the MDM2 gene [48]. To model reaction 5, we have used a Hill function, which accounts for the number of p53 molecules required to induce gene expression. Furthermore, based on [2] and [13], we have assumed that $r_{5}$ is an increasing function of the amount of DNA damage.

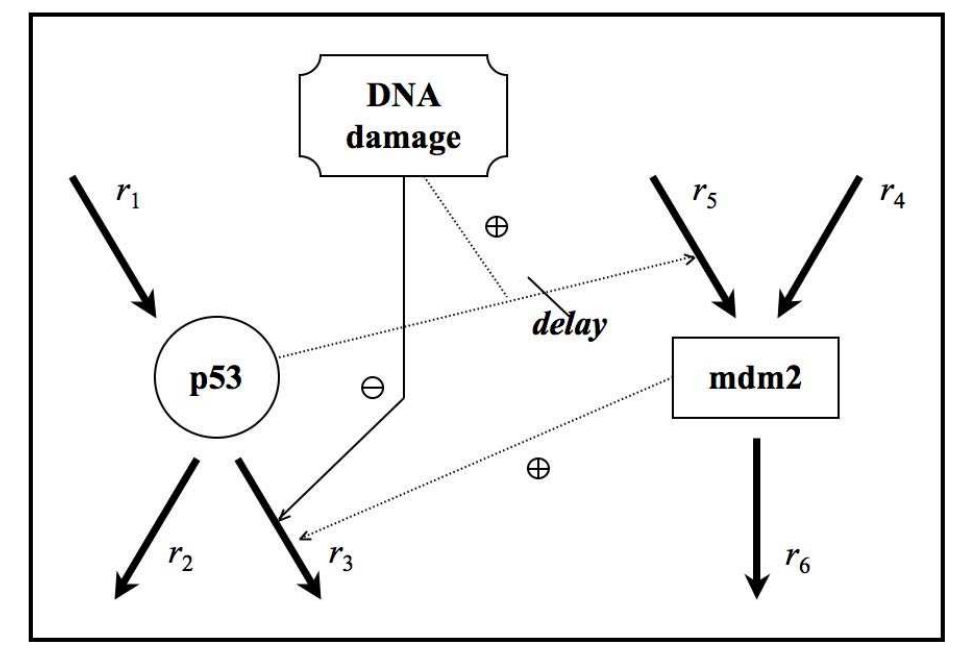

Figure 1: Schematic representation of our kinetic model for the interactions between p53 and mdm2. Bold arrows represent protein synthesis and elimination processes, which can be promoted (dotted lines) or inhibited (solid lines) by the components of the system. For $i=1, \ldots, 6$, the symbol $r_{i}$ denotes the rate of reaction $i$.

To prevent the simple network (Fig.1) from immediately inhibiting itself and, in particular, to enable oscillations to occur, a delay in the negative feedback loop is required [42]. Such a delay can be modelled in several ways. In some cases the delay has been modelled implicitly - [18], for instance, included an additional unknown component in the p53-mdm2 pathway, whereas [5] combined positive and negative feedbacks. The most common approach, however, is to incorporate 
time delays explicitly and this has several uses in terms of modelling intracellular processes. [40] showed that discrete delay terms can help reduce the number of variables and parameters required to describe a molecular system by replacing one or more intermediate reactions. Concerning spatial effects, [26] demonstrated that the waiting times associated with transcription and translation can be fused into a single time delay without altering the dynamical properties of the system. Hence, [31] modelled the rate of mdm 2 synthesis at time $t$ as a function of the amount of p53 present in the system at time $t-\tau$. [46] used delay terms to describe mRNA translation and protein transport processes within the Wnt signalling pathway. Therefore in line with this previous work, here we also use a time delay term to model p53-induced DNA transcription, nuclear export of mRNA, and cytoplasmic translation processes. In particular, we use a so-called distributed time delay in which the amount of mdm 2 produced at time $t$ depends on the abundance of $\mathrm{p} 53$ within the preceding time interval, which is expressed as

$$
\int_{-\infty}^{t} w_{p}(t-s)[P(s)] \mathrm{d} s
$$

where $[P(u)]$ is the concentration of $\mathrm{p} 53$ at time $u$ and $w_{p}(u)=\mathrm{e}^{-u}$ is a weighting function that assigns less transactivation potential to those p53 molecules present a longer time ago.

Given the assumptions above, the reaction rates can be calculated as follows:

$$
\begin{aligned}
& r_{1}(t)=s_{p}, \\
& r_{2}(t)=d_{p 1}[P], \\
& r_{3}(t)=\frac{d_{p 2}[M][P]}{K_{1}+Z}, \\
& r_{4}(t)=s_{m 1}, \\
& r_{5}(t)=\left(s_{m 2}+\frac{s_{m 3} Z}{K_{2}+Z}\right) \frac{\left(\int_{-\infty}^{t} w_{p}(t-s)[P(s)] \mathrm{d} s\right)^{h}}{K_{3}+\left(\int_{-\infty}^{t} w_{p}(t-s)[P(s)] \mathrm{d} s\right)^{h}}, \\
& r_{6}(t)=d_{m}[M],
\end{aligned}
$$

where $[M]$ and $[P]$ represent the concentrations of mdm2 and p53, $s_{\star}$ and $d_{\star}$ are constant synthesis and degradation rates, and the parameters $K_{\star}$ are saturation constants. The Hill exponent $h$ denotes the number of p53 molecules involved in inducing transcription of the MDM2 gene. Finally, the parameter $Z$ denotes the amount of DNA damage. Hence from (2.1), (2.2), (2.3), and (2.4), the system of ODEs describing our $\mathrm{p} 53-\mathrm{mdm} 2$ dynamics is given by

$$
\begin{aligned}
& \frac{\mathrm{d}[P]}{\mathrm{d} t}=s_{p}-\left(d_{p 1}+\frac{d_{p 2}[M]}{K_{1}+Z}\right)[P], \\
& \frac{\mathrm{d}[M]}{\mathrm{d} t}=s_{m 1}+\left(s_{m 2}+\frac{s_{m 3} Z}{K_{2}+Z}\right) \frac{\left(\int_{-\infty}^{t} w_{p}(t-s)[P(s)] \mathrm{d} s\right)^{h}}{K_{3}+\left(\int_{-\infty}^{t} w_{p}(t-s)[P(s)] \mathrm{d} s\right)^{h}}-d_{m}[M],
\end{aligned}
$$

with initial conditions $P\left(t_{0}\right)=P_{0},\left[M\left(t_{0}\right)\right]=M_{0}$. 
In order to make both analytical and numerical progress, we make use of the functional form of the distributed time delay which enables us to re-write our equations without a delay term. First we define:

$$
F_{p}(t)=\int_{-\infty}^{t} w_{p}(t-s)[P(s)] \mathrm{d} s .
$$

Following [3] we then multiply (2.7) by $\mathrm{e}^{t}$ and differentiate with respect to $t$ :

$$
\frac{\mathrm{d}}{\mathrm{d} t}\left(F_{p}(t) \mathrm{e}^{t}\right)=\frac{\mathrm{d}}{\mathrm{d} t} \int_{-\infty}^{t} \mathrm{e}^{s}[P(s)] \mathrm{d} s,
$$

which yields

$$
\left(\frac{\mathrm{d} F_{p}}{\mathrm{~d} t}+F_{p}(t)\right) \mathrm{e}^{t}=\mathrm{e}^{t}[P(t)]
$$

and, thus:

$$
\frac{\mathrm{d} F_{p}}{\mathrm{~d} t}=[P(t)]-F_{p}(t)
$$

We may thus replace the distributed delay term by an additional ordinary differential equation which facilitates the analysis, numerical analysis and spatial extension of our model enormously. Hence the final system of ordinary differential equations (ODEs) equivalent to (2.5) and (2.6) is given by:

$$
\begin{aligned}
& \frac{\mathrm{d}[P]}{\mathrm{d} t}=s_{p}-\left(d_{p 1}+\frac{d_{p 2}[M]}{K_{1}+Z}\right)[P], \\
& \frac{\mathrm{d}[M]}{\mathrm{d} t}=s_{m 1}+\left(s_{m 2}+\frac{s_{m 3} Z}{K_{2}+Z}\right) \frac{F_{p}^{h}}{K_{3}+F_{p}^{h}}-d_{m}[M], \\
& \frac{\mathrm{d} F_{p}}{\mathrm{~d} t}=[P]-F_{p},
\end{aligned}
$$

with initial conditions $P\left(t_{0}\right)=P_{0},\left[M\left(t_{0}\right)\right]=M_{0}$ and $F_{p}\left(t_{0}\right)=F_{p 0}$. It can be shown (Appendix) that for low $Z$ values the system (2.8) reaches a stable steady state. For higher $Z$ values, however, it can give rise to a stable limit cycle (Fig.2). The parameter values used in the model simulations are provided in the Appendix (Table A.1).

\section{The spatial p53-mdm2 model}

A natural, but till now unexplored, next step in p53-mdm 2 modelling is to consider the spatial location and diffusion of the proteins within a cell. For this purpose, we considered a two-dimensional cell with a circular nucleus and modified (2.1) and (2.2) to transform them into partial differential equations (PDEs):

$$
\begin{aligned}
& \frac{\partial[P]}{\partial t}=\bar{r}_{1}-\bar{r}_{2}-\bar{r}_{3}+D_{p} \nabla^{2}[P], \\
& \frac{\partial[M]}{\partial t}=\bar{r}_{4}+\bar{r}_{5}-\bar{r}_{6}+D_{m} \nabla^{2}[M],
\end{aligned}
$$




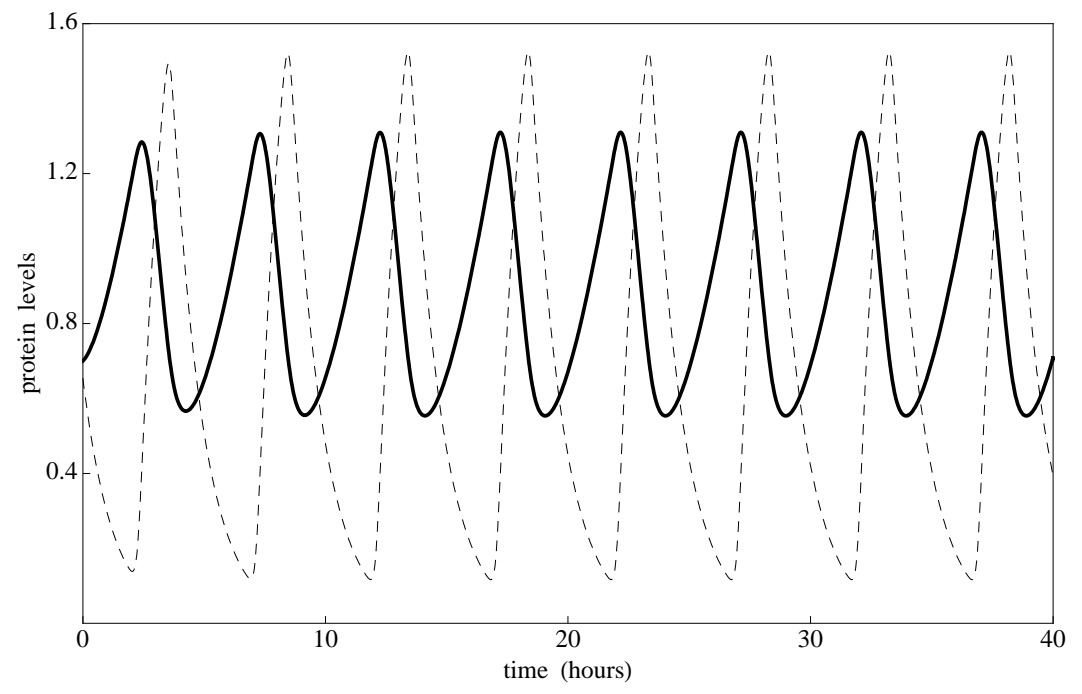

Figure 2: Sustained oscillations of p53 (solid line) and mdm2 (broken line) in response to a DNA damage level of $Z=1$. Simulations performed with (2.8).

where $[M],[P]$ and the $\bar{r}_{i}$ 's are now functions of position as well as time. We scaled the model by choosing a length scale of $25 \mu \mathrm{m}$ and a timescale of one hour (i.e. the non-dimensional length and time units correspond to $25 \mu \mathrm{m}$ and one hour respectively). The origin, $(x, y)=(0,0)$, corresponds to the centre of the nucleus. The parameters $D_{p}$ and $D_{m}$ are non-dimensional diffusion coefficients for p53 and mdm2, respectively. In addition to including diffusion terms, we adjusted the expressions for the protein synthesis rates to account for spatio-temporal variation. As proteins are produced by ribosomes, which are often more ubiquitous in the proximity of the nucleus, we assumed that $\bar{r}_{1}, \bar{r}_{4}$ and $\bar{r}_{5}$ decrease as the distance from the nucleus increases. We modelled this by multiplying the synthesis rates by a weighting function,

$$
\begin{aligned}
& w_{s}(x, y) \quad=\left(1-\tanh \left(\sqrt{x^{2}+y^{2}}-\psi_{n}\right)\right) \quad \text { in the cytoplasm, } \\
& w_{s}(x, y) \quad=0 \quad \text { in the nucleus, }
\end{aligned}
$$

with $\psi_{n}$ is the non-dimensional radius of the nucleus. According to this definition, the weighting function reaches its highest values close to the nucleus. Figure 3 illustrates the shape of $w_{s}$ for a circular cell (diameter $\Phi_{c}=2$ ) with a central circular nucleus (radius $\psi_{n}=0.4$ ). As before we model the p53-induced DNA transcription, nuclear export of mRNA, and cytoplasmic translation with our delay terms [19, 26, 40,47]. Hence, the resulting system of PDEs is 


$$
\begin{aligned}
\frac{\partial[P]}{\partial t}= & D_{p}\left(\frac{\partial^{2}[P]}{\partial x^{2}}+\frac{\partial^{2}[P]}{\partial y^{2}}\right)+w_{s}(x, y) s_{P}-\left(d_{p 1}+\frac{d_{p 2}[M]}{K_{1}+Z}\right)[P] \\
\frac{\partial[M]}{\partial t}= & D_{m}\left(\frac{\partial^{2}[M]}{\partial x^{2}}+\frac{\partial^{2}[M]}{\partial y^{2}}\right)+w_{s}(x, y) s_{m 1}-d_{m}[M] \\
& +w_{s}(x, y)\left(s_{m 2}+s_{m 3} \frac{Z}{K_{2}+Z}\right) \frac{\left(\int_{-\infty}^{t} w_{p}(t-s)[P(., s)] \mathrm{d} s\right)^{h}}{K_{3}+\left(\int_{-\infty}^{t} w_{p}(t-s)[P(., s)] \mathrm{d} s\right)^{h}}
\end{aligned}
$$

with initial conditions $[P](x, y, 0)=[M](x, y, 0)$ and zero-flux boundary conditions.

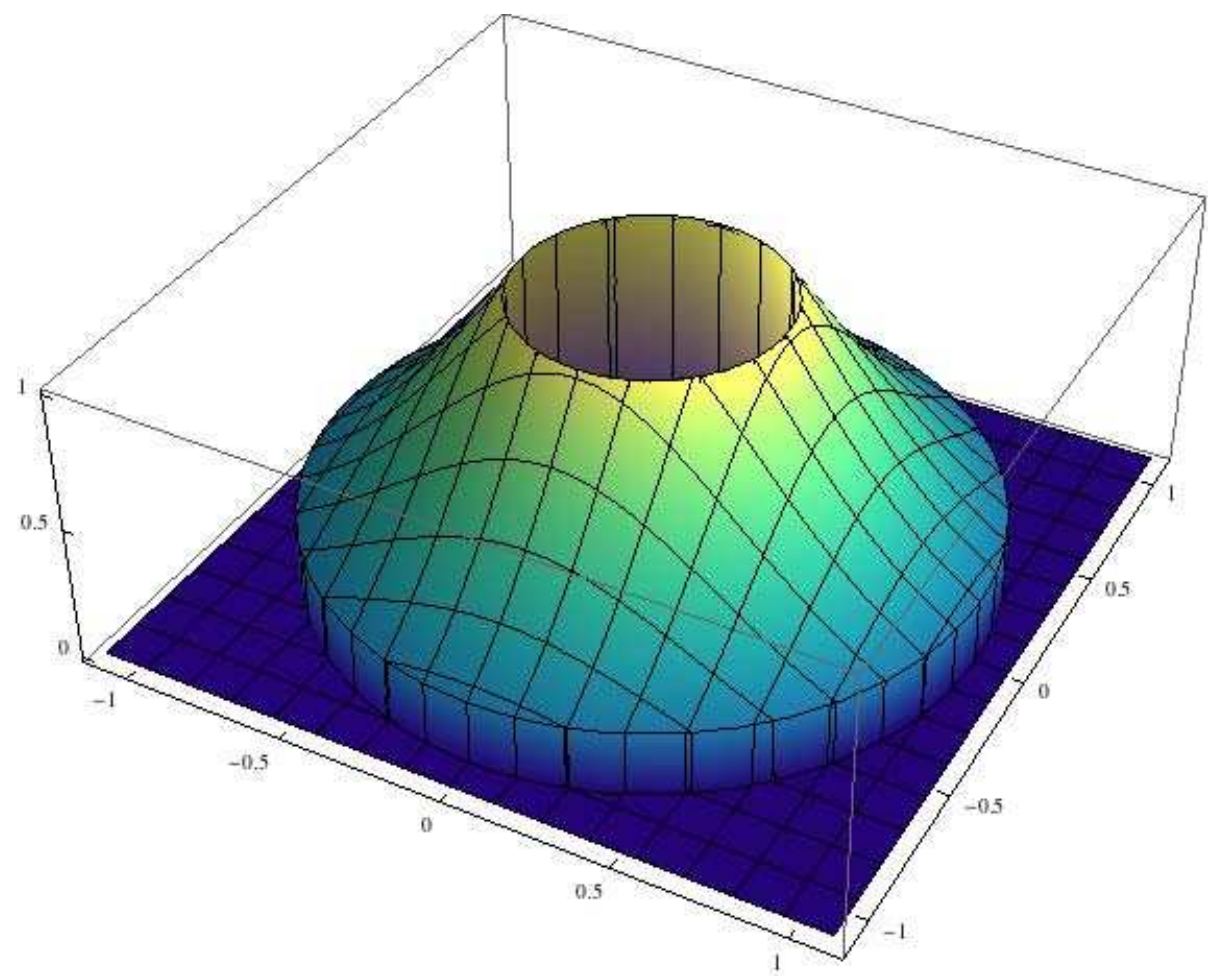

Figure 3: Protein synthesis weighting function. Circular cell with a central circular nucleus. Dimensionless parameter values: $\Phi_{c}=2$ and $\psi_{n}=0.4$ (i.e. cell diameter $=50 \mu \mathrm{m}$; diameter of the nucleus $=20 \mu \mathrm{m})$. 
Once again in order to simplify the computational simulations, using (2.7) and the technique of [3], we replace the above system without the delay terms as follows:

$$
\begin{aligned}
\frac{\partial[P]}{\partial t}= & D_{p}\left(\frac{\partial^{2}[P]}{\partial x^{2}}+\frac{\partial^{2}[P]}{\partial y^{2}}\right)+w_{s}(x, y) s_{P}-\left(d_{p 1}+\frac{d_{p 2}[M]}{K_{1}+Z}\right)[P], \\
\frac{\partial[M]}{\partial t}= & D_{m}\left(\frac{\partial^{2}[M]}{\partial x^{2}}+\frac{\partial^{2}[M]}{\partial y^{2}}\right)+w_{s}(x, y) s_{m 1}-d_{m}[M] \\
& +w_{s}(x, y)\left(s_{m 2}+s_{m 3} \frac{Z}{K_{2}+Z}\right) \frac{F_{p}^{h}}{K_{3}+F_{p}^{h}}, \\
\frac{\partial F_{p}}{\partial t}= & {[P]-F_{p}, }
\end{aligned}
$$

with initial conditions $[P](x, y, 0)=[M](x, y, 0)=F_{p}(x, y, 0)=0$ and zero-flux boundary conditions. The numerical simulations shown below were produced using the COMSOL/FEMLAB ${ }^{\dagger}$ package, which uses the finite element technique [49]. Triangular basis elements and Lagrange quadratic basis functions along with a backward Euler time-stepping method for integrating the equations were used in all simulations.

\section{Results}

To study the impact of cell shape and position of the nucleus on the spatio-temporal dynamics of the network shown in Fig.1, we considered a two-dimensional domain with a circular subdomain (or nucleus). First, we solved the system (3.6) for a circular cell that has the nucleus located in its centre. Snap-shots of the solution for the concentration of p53 at four different time points are shown in Fig.4. The simulations emulate an intracellular response to a DNA damage level of $Z=1$ (hence guaranteeing a limit cycle and therefore oscillatory kinetics, see below). Initially, high concentration waves emerge near the nucleus, from where they move outwards (Fig.4.a and $b$ ). Consequently, the p53 level oscillates at each fixed point in space. As time evolves, the concentric rings begin to break up, giving rise to irregular waves, and the patterns gradually increase in complexity (Fig.4.c and $d$ ). The solution for mdm2 behaves similarly (not shown). These complex, heterogeneous spatio-temporal dynamics are the result of the combination of diffusion with the limit-cycle kinetics. Qualitatively similar dynamics have been observed previously in ecological models (cf. [33, 36, 37, 38]).

Second, we solved the same system for another circular domain with its nucleus off-centre. Fig.5 depicts the results for p53. Initially, we observed a propagation of concentric high-density rings similar to that in Fig.4.b. However, in Fig.5 the circular waves reach the cell's boundaries

\footnotetext{
†http://www. femlab.com/
} 

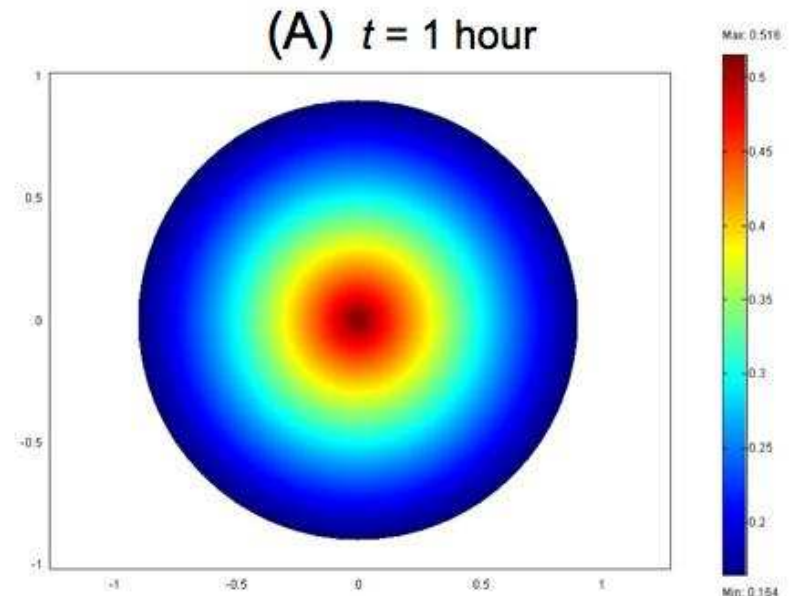

(B) $t=9$ hours
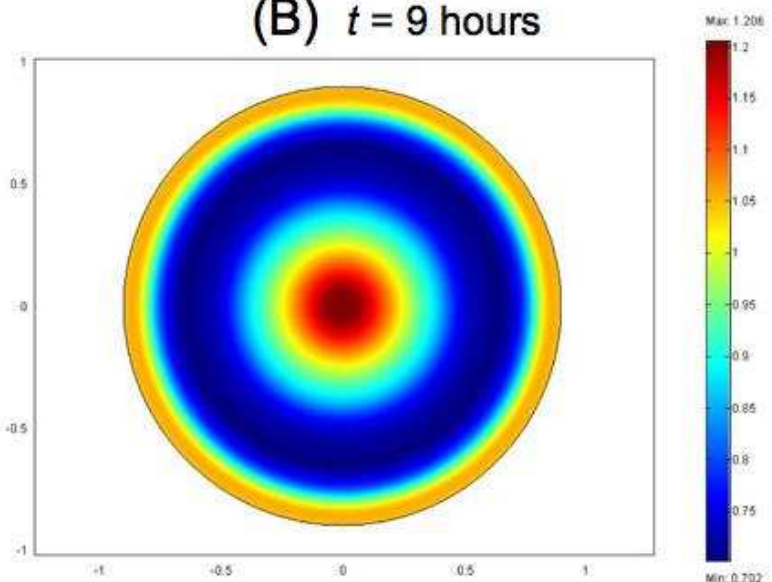

(C) $t=230$ hours
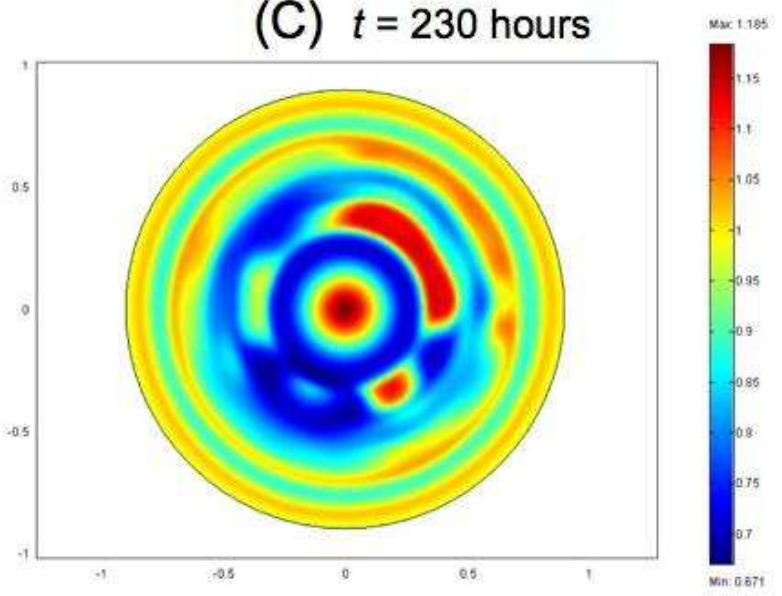

(D) $t=400$ hours

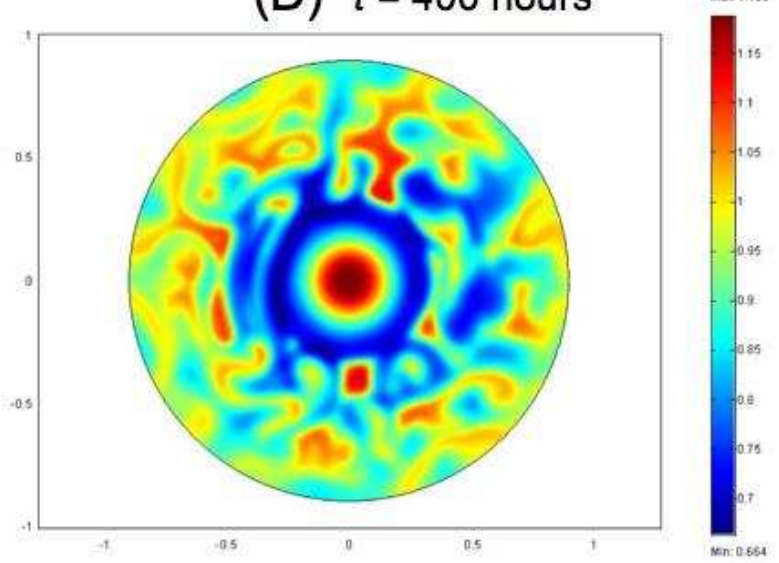

Figure 4: Snap-shots of the p53 concentration in a circular domain with a central circular nucleus taken at four different times during the same simulation. Parameter values: $\Phi_{c}=2, \psi_{n}=0.1$ and $Z=1$. Simulations performed with (3.6). 

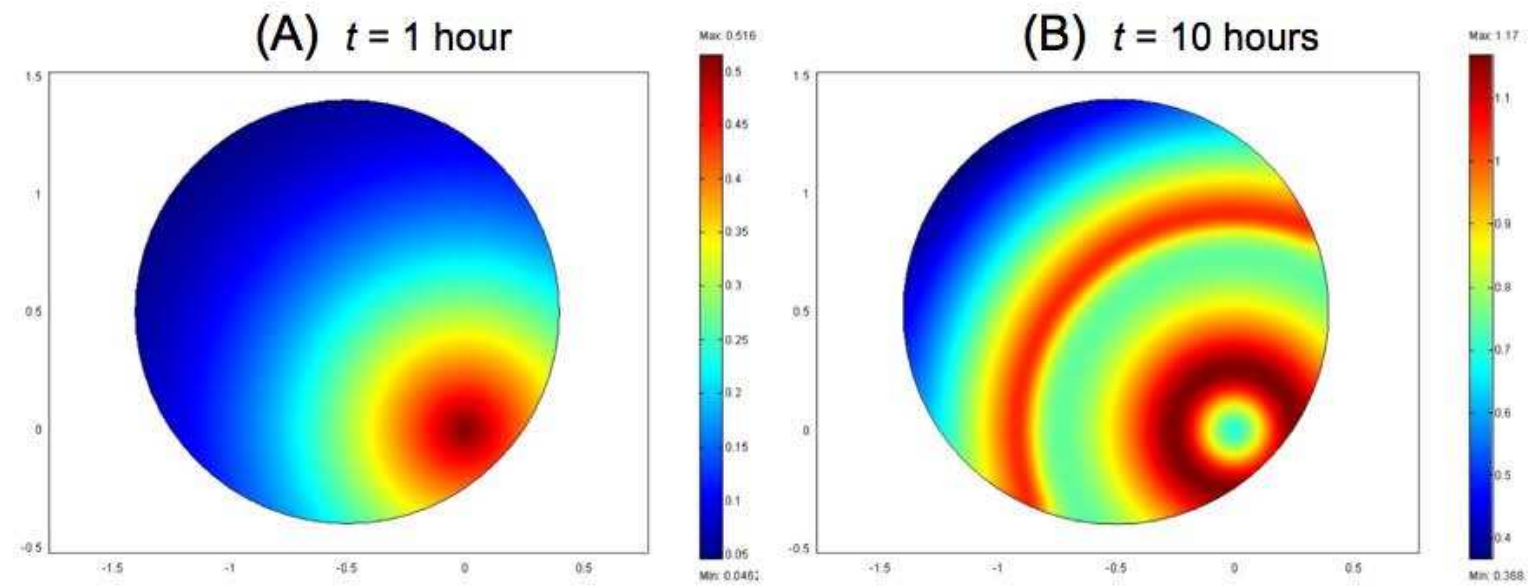

(C) $t=140$ hours

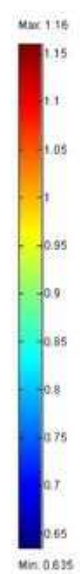

(D) $t=400$ hours
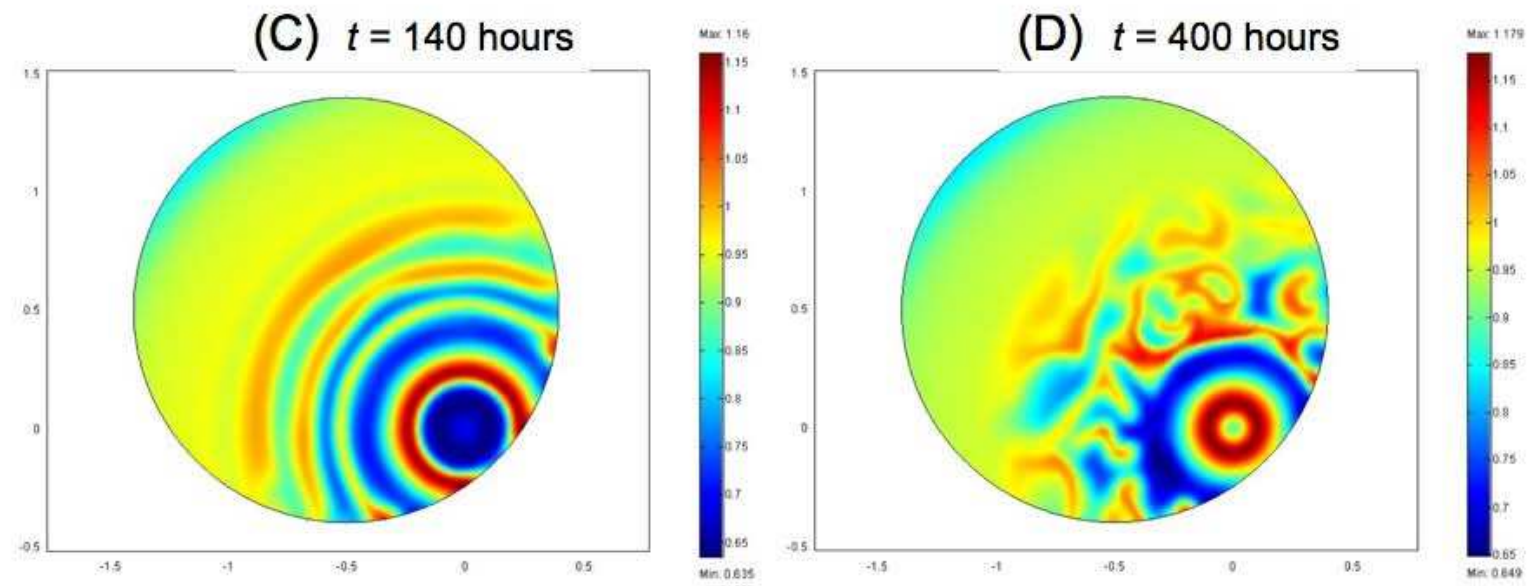

Figure 5: Snap-shots of the p53 concentration taken at four different times during the simulation. Circular cell with a circular off-centre nucleus. Parameter values: $\Phi_{c}=2, \psi_{n}=0.1$ and $Z=1$. Simulations performed with (3.6). 
quicker than in Fig.4 and, consequently, symmetry is lost sooner. Analogous early wave breakdown was also seen in noncircular domains. This prediction is exemplified in Fig.6 for the spatiotemporal dynamics of p53 within an elliptical cell with a central circular nucleus. To facilitate comparison of Fig. 6 with Figs. 4 and 5, we have chosen the length of the semiaxes such that the area of the resulting ellipse is equal to that of a circle of diameter $\Phi_{c}=2$.
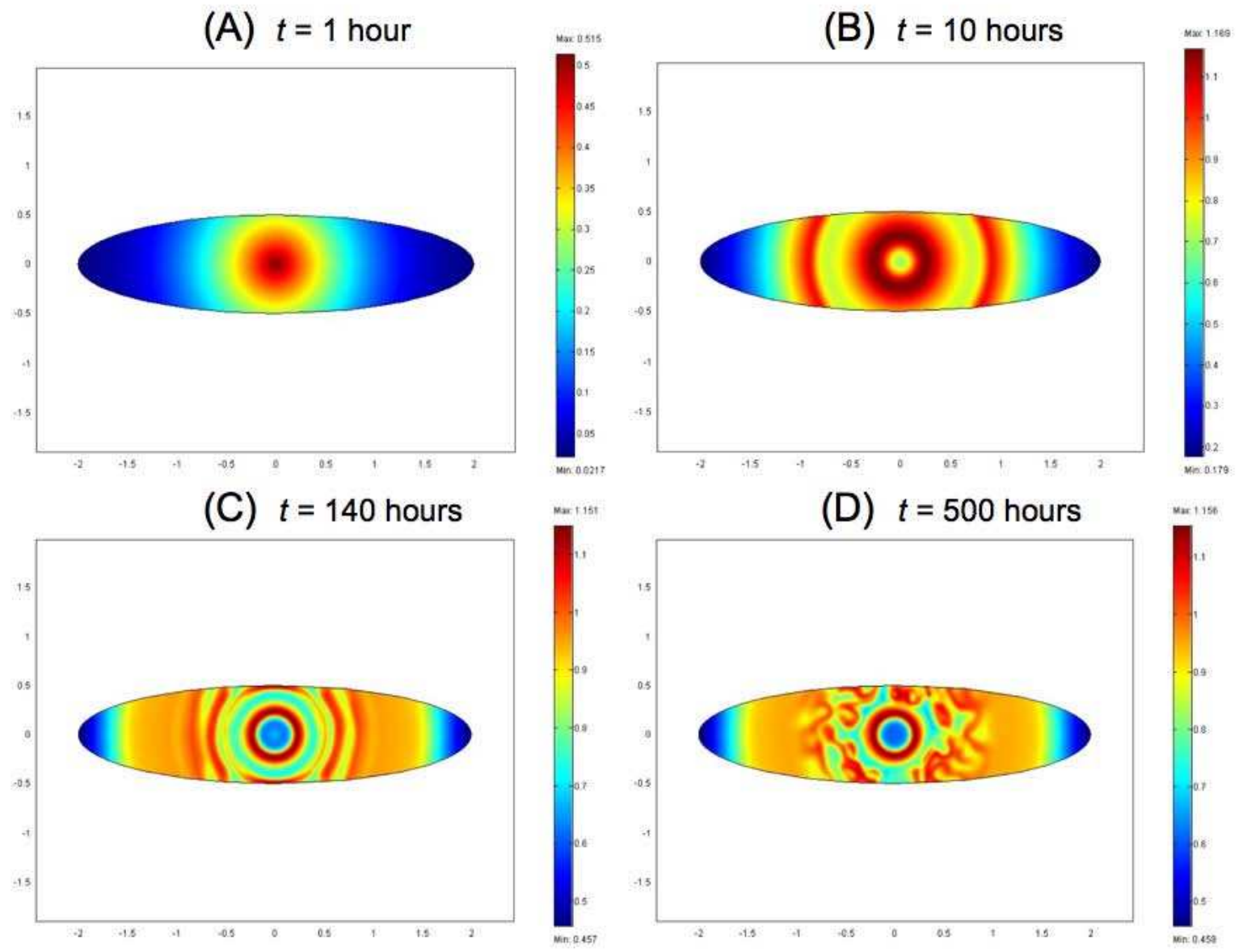

Figure 6: Snap-shots of the p53 concentration taken at four different times during the simulation. Elliptical cell with a central, circular nucleus. The domain has the same surface area as the cells shown in Figs 4 and 5. Length of semimajor axis $=2.0$, length of semiminor axis $=0.5, \psi_{n}=0.1$ and $Z=1$. Simulations performed with (3.6).

Finally (Figs.7 and 8), we investigated the temporal dynamics of the total amounts of protein, which we obtained by integrating over the 2D domain. In Fig.7.a we show the changes in total p53 and $\mathrm{mdm} 2$ in response to DNA damage for various cell morphologies. According to our model 
simulations, the average total concentration can depend on the shape of the domain as well as on the position of the nucleus. It is important to note, however, that these differences might be partly due to spatial effects on the overall protein synthesis rate. Regarding the temporal changes in the total p53 concentration, we predict that high amplitude oscillations are more common in circular domains with central nuclei, as this particular morphology facilitates the formation of concentric high-density rings. Low amplitude oscillations take place when the outer waves break against the cells boundaries.

In Fig.7.b we compare p53's response to different levels of stress in a circular cell with a central nucleus. For the nonspatial model (2.8), it can be shown that the solutions pass through a Hopf bifurcation at $Z=0.323$, leaving a stable steady state to enter a limit cycle (Appendix). Periodic solutions continue till $Z=4.725$, where the unstable steady state becomes stable again. Adding diffusion terms to (2.1-2.2) to obtain (3.1-3.2) does not alter these bifurcations points. Incorporating the spatial variation in protein synthesis (Fig.3), however, causes an increase in the amount of DNA damage required to induce sustained p53 oscillations. Hence, for the domain in Figs. 4 and $7 . b$ only values of $Z$ greater than 0.494 result in a limit cycle.

In addition to varying the level of DNA damage, we also studied the impact of the diffusion rates on the behaviour of the total levels of protein. The two panels in Fig.8 show our model predictions under slow and fast diffusion conditions, respectively. These simulations were performed with (3.6) for a circular domain with a central nucleus exposed to DNA damage level of $Z=1$. When the diffusion coefficients are small (Fig.8-left), the total levels of protein oscillate with high frequency but low amplitude. As the diffusion coefficients increase (Fig.8-right), however, the amplitude of the oscillations becomes more variable and, in particular, larger scale oscillations become more common. This occurs because fast diffusion promotes the formation of high-density waves within the domain, which translate in high amplitude oscillations in the total protein level. Hence, each high peak in total p53 (Fig.8) means that a new high-density ring has appeared around the nucleus while a previous high-density ring is still present. In summary, if the diffusion of p53 and $\mathrm{mdm} 2$ is fast, concentric rings move through the domain without colliding, whereas if protein diffusion is slow, wave collision occurs and more complicated density patterns arise.

\section{Discussion}

A plethora of mathematical models have been proposed to describe various molecular pathways. Most models provide a system of ordinary differential equations (ODEs), describing the changes in the total concentrations of various proteins, which implicitly assume a well-stirred, homogeneous solution. [24] have, however, recently suggested that morphological and spatial information are crucial in determining the behaviour and function of intracellular pathways. In particular, they showed that signalling networks could be switched on or off within different domains and subdomains, depending on their size and shape. "The cell itself is the corresponding protein pattern- 

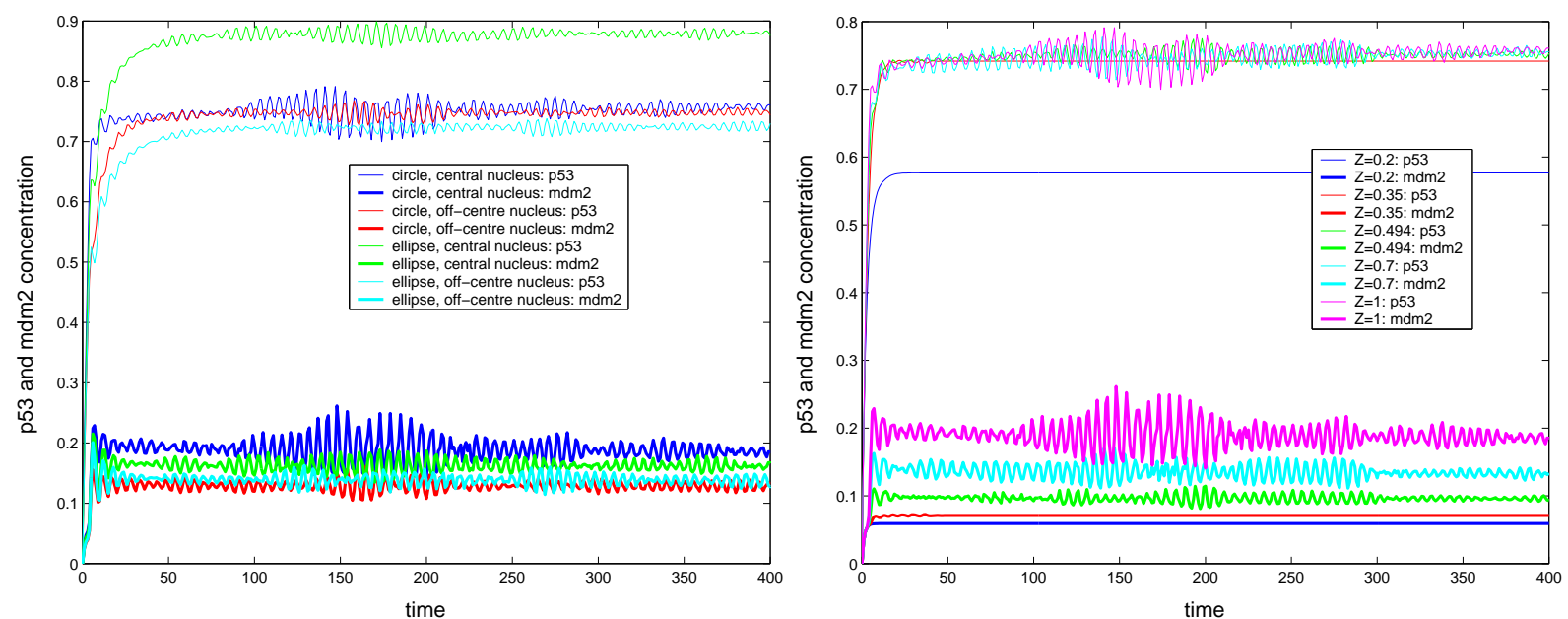

Figure 7: Total protein concentrations as a function of time. Simulations performed with (3.6). (Left) Total levels of mdm2 (bold lines) and p53 for different cell morphologies. DNA damage, $Z=1$. (Right) Total concentrations of mdm2 (bold lines) and $\mathrm{p} 53$ in response to different levels of DNA damage; simulations for a circular cell with a central circular nucleus.
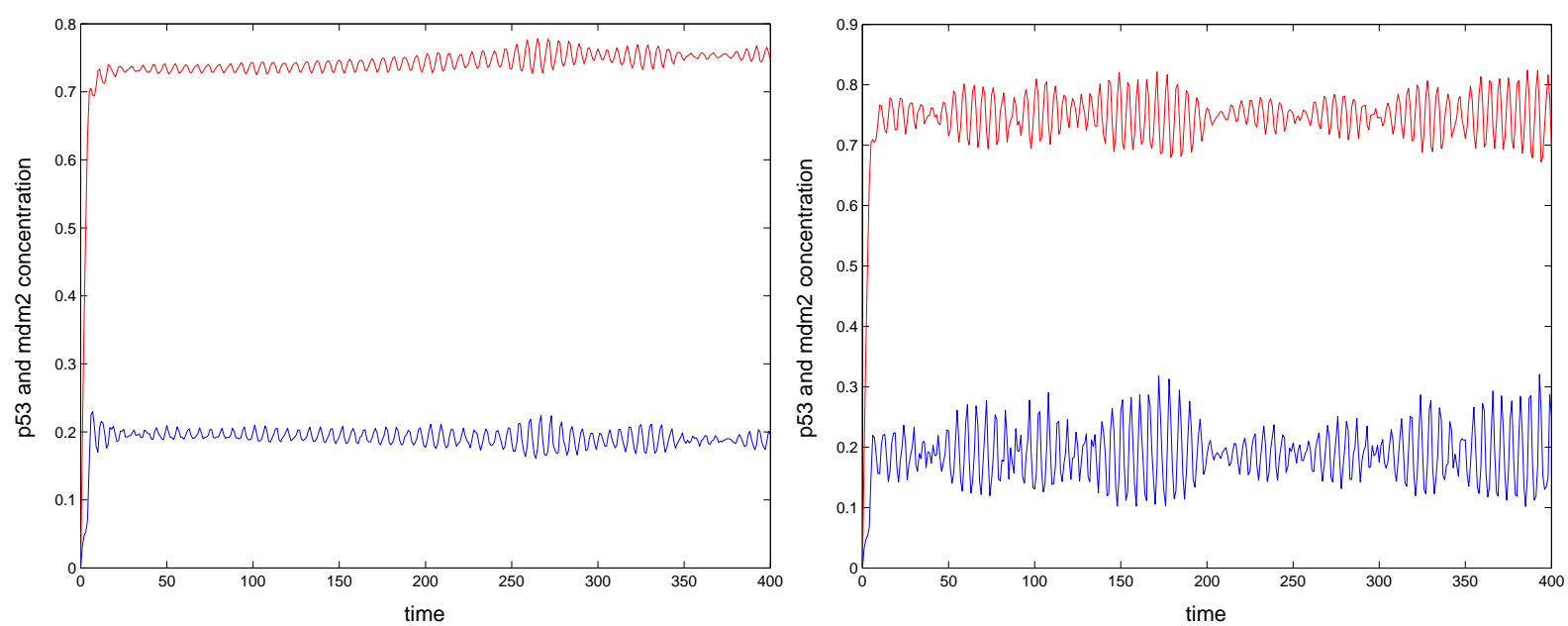

Figure 8: Total concentrations of p53 (red) and mdm2 (blue) as a function of time. Simulations performed with (3.6) for a circular domain with a central nucleus (Fig.4). Parameter values: $\Phi_{c}=$ $2, \psi_{n}=0.1$ and $Z=1$. (Left) $D_{p}=D_{m}=10^{-5}$. (Right) $D_{p}=D_{m}=10^{-3}$. 
formation apparatus or protein co-compartmentalization machine, thus, each protein must be at the right time, at the right location, and at the right concentration in a cell to interact with other proteins" [35]. Here we have presented a theoretical study of the influence of spatial effects on the dynamics of a subclass of networks characterised by the presence of at least one negative feedback loop and a transcriptional delay. We used a minimal, representative model of the p53 pathway (Fig.1) that is able to demonstrate oscillatory behaviour under certain conditions. Our model analysis and numerical simulations revealed that the amplitude and frequency of the oscillations can strongly depend on both the shape of the cell and the position of the nucleus.

The model also provides a new interpretation of experimentally observed digital pulses in p53 levels in single cells [17]. Our simulations showed that, for certain parameter values, alternate sequences of high- and low-amplitude oscillations occur. We propose that the discrete peaks may correspond to snap-shots of our high-amplitude sequences. This hypothesis is depicted in Fig.9. Shorter waiting-times between subsequent time-lapse fluorescence microscopy images in combination with lower detection thresholds may reveal the high-frequency oscillations of variable amplitude suggested by our spatial model.

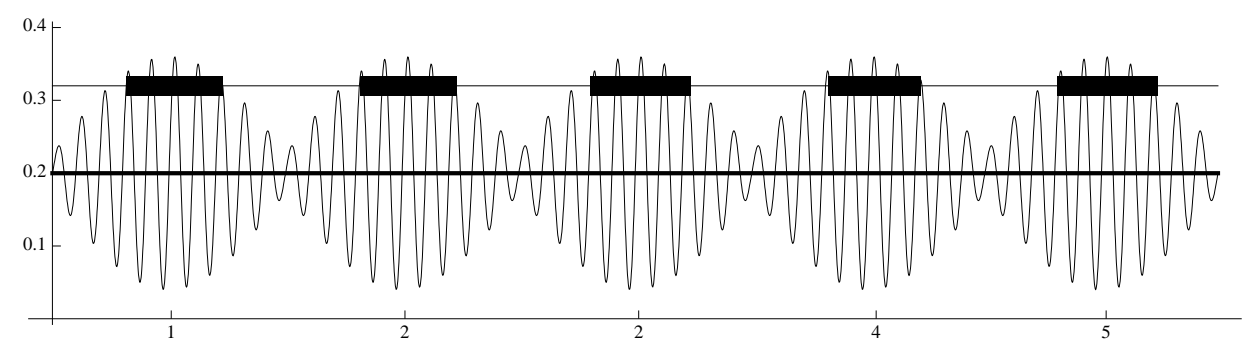

Figure 9: Schematic showing how a sequence of high and low amplitude oscillations can give rise to digital pulses. The total protein concentration oscillates in response to DNA damage. The lower and upper horizontal lines represent the average concentration and the protein's detection threshold, respectively. The black boxes highlight the discrete peaks predicted for the given threshold.

While building a comprehensive spatio-temporal model for the p53 pathway, our approach has inevitably oversimplified several aspects of this regulatory network. A natural direction for extending the work presented in this paper is therefore to include additional biological knowledge. Below we briefly discuss several possibilities. Firstly, alternative expressions for the protein synthesis weighting function may be considered. With the definition given in equation (3.3), the total protein synthesis rates depend on both the shape of the domain and position of the nucleus, making the protein dynamics taking place in different domains hard to compare. Secondly, the processes of p53-induced DNA transcription, nuclear export of mRNA, and cytoplasmic translation could be modelled explicitly rather than using delay terms. Finally, additional molecular components and phenomena may be incorporated into Fig.1, such as the dynamics of the mdm2-p53 complex, the role of the Arf tumour suppressor [10], the regulation of active nuclear protein export $[9,30]$, and the effect of various post-transcriptional modifications (e.g. [2, 41]). A more detailed 
kinetic network would have the advantage of model parameters having a well-defined biochemical interpretation. Hence, developing such a model would enable us not only to identify, but also to measure key parameters that have a strong impact on the behaviour of the system. Such a set of parameter values might provide a better agreement between the predicted and observed p53 peak frequencies $[17,18]$. Recent advances in topological proteomics suggest that spatial information on the subcellular localisation of proteins and processes will soon become available, enabling us to further parameterise, calibrate and validate our models. Hence, using a new robotic imaging technology known as Multi-Epitope-Ligand Kartographie (MELK), it is already possible to map entire protein networks in morphologically intact cells and tissues [32, 35].

\section{Acknowledgements}

The authors gratefully acknowledge various funding sources. KEG was supported by an EPSRC Mathematics CASE PhD studentship. IMMvL is funded through a Discipline Hopping Award (MRC/EPSRC). SL thanks the EC for financial support through the FP6 OPAALS project. The work of MAJC was supported by a Leverhulme Trust Personal Research Fellowship. This publication reflects only the authors' views. The EC is not liable for any use that may be made of the information therein.

\section{APPENDIX}

\section{Stability analysis of the kinetic model}

The Jacobian matrix of system (2.8) has the form:

$$
\left(\begin{array}{ccc}
-a & 0 & d \\
-e & -b & 0 \\
0 & f & -c
\end{array}\right)
$$

where $a=d_{m}, b=\left(d_{p 1}+\frac{d_{p 2}[M]^{\star}}{K_{1}+Z}\right), c=1, d=\left(s_{m 2}+s_{m 3} \frac{Z}{K_{2}+Z}\right) \frac{h K_{3}\left(F_{p}^{\star}\right)^{h-1}}{\left(K_{3}+\left(F_{p}^{\star}\right)^{h}\right)^{2}}, e=\frac{d_{p 2}[P]^{\star}}{K_{1}+Z}$, and $f=1$, with $[P]^{\star},[M]^{\star}$ and $F_{p}^{\star}$ a steady state solution of (2.8). The eigenvalues can be found by solving:

$$
\theta(\lambda)=\lambda^{3}+\alpha \lambda^{2}+\beta \lambda+\gamma=0
$$

where $\alpha=a+b+c, \beta=a b+b c+c a$ and $\gamma=a b c+d e f$. For characteristic equations of this form, the Routh-Hurwitz theorem states that the steady steate is stable if and only if $\alpha>0, \gamma>0$, and $\alpha \beta>\gamma$ [8]. As $a, b, c, d, e, f>0$, the first two conditions are satisfied. The third condition implies that the steady state is stable if and only if

$$
(a+b+c)(a b+b c+c a)>a b c+d e f .
$$


Given the parameter values in Table A.1, we have investigated the behaviour of the solutions as a function of DNA damage (Fig.10). Hopf bifurcations and bifurcation diagrams were obtained using the AUTO tool ${ }^{\ddagger}$ within XPP [7].

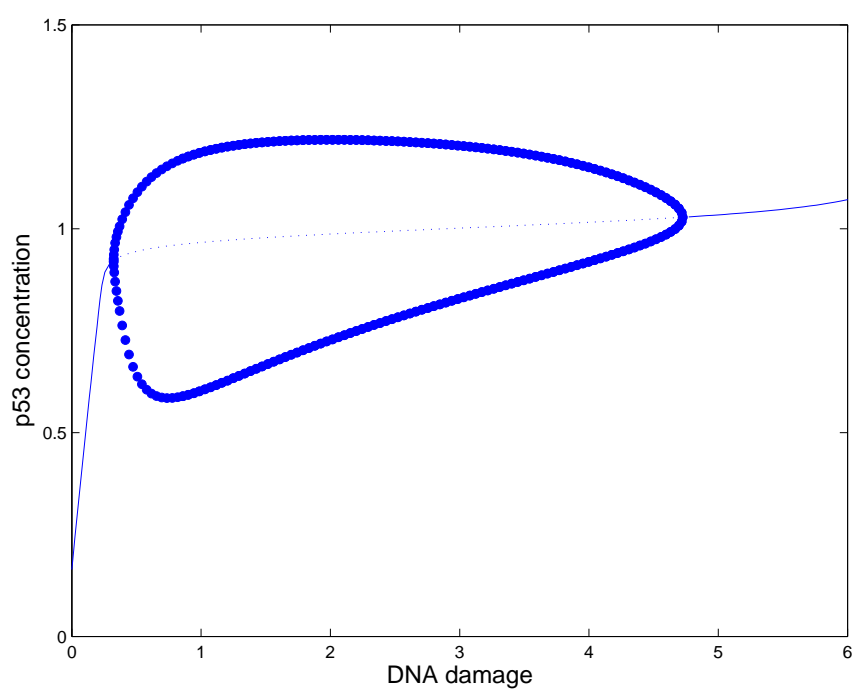

Figure 10: Bifurcation diagram for the concentration of p53 against DNA damage. System (2.8) with the parameter values from Table A.1. The solid and broken lines represent a stable and unstable steady states, respectively. The bullet points correspond to stable periodic solutions.

\section{Parameter values}

As we were unable to find diffusion coefficients for p53 and mdm2 in the literature, we chose values for $D_{p}$ and $D_{m}$ based on the propagation rates of other proteins, such as:

- Green fluorescent protein: $D=0.13 \mu \mathrm{m}^{2} \mathrm{~s}^{-1}$ in the plasma membrane of E.coli [27].

- E-cadherin: $D=0.00092 \mu \mathrm{m}^{2} \mathrm{~s}^{-1}$ when the molecules follow simple Brownian motion [16].

In our simulations we used $D=0.00002 \mu \mathrm{m}^{2} \mathrm{~s}^{-1}$ (i.e. $D_{p}=D_{m}=0.0001$ ).

\section{References}

[1] B. Alberts, A. Johnson, J. Lewis, K. Roberts, P. Walter. Molecular Biology of the Cell, Fourth Edition. Garland Science, Taylor and Francis Group Ltd, Oxford, 2002.

[2] E. Appella, C.W. Anderson. Post-transcriptional modifications and activation of p53 by genotoxic stresses. Eur. J. Biochem., 268 (2001), 2764-2772.

\footnotetext{
${ }^{\ddagger}$ http://indy.cs.concordia.ca/auto/
} 
Table 1: Parameter values. The two values shown in the third column correspond to nondimensionalised and original parameters, respectively. Sources: $(a)[1,20] ;(b)[18] ;(c)[16,27] ;(d)[23]$; and $(e)$ best guess.

\begin{tabular}{|c|l|ll|}
\hline Symbol & Interpretation & Value & $(a)$ \\
\hline$\Phi_{c}$ & Cell diameter & $2(50 \mu \mathrm{m})$ & $(a)$ \\
$\psi_{n}$ & Radius of the nucleus & $0.1(2.5 \mu \mathrm{m})$ & $(e)$ \\
$d_{m}$ & mdm2 degradation rate & 0.8 & $(b)$ \\
$d_{p 1}$ & mdm2-independent p53 degradation rate & $2.5 e^{-04}$ & $(e)$ \\
$d_{p 2}$ & p53 degradation coefficient & 1 & $(c)$ \\
$D_{m}$ & mdm2 diffusion coefficient & $0.0001\left(0.00002 \mu \mathrm{m}^{2} \mathrm{~s}^{-1}\right)$ & $(c)$ \\
$D_{p}$ & p53 diffusion coeficient & $0.0001\left(0.00002 \mu \mathrm{m}^{2} \mathrm{~s}^{-1}\right)$ & $(b)$ \\
$h$ & Hill exponent & 50 & $(e)$ \\
$K_{1}$ & Saturation coefficient p53 degradation & 0.05 & $(e)$ \\
$K_{2}$ & mdm2 synthesis saturation coefficient & 0.05 & $(b)$ \\
$K_{3}$ & Hill saturation coefficient & 1.066 & $(b)$ \\
$s_{m 1}$ & p53-independent mdm2 synthesis rate & $2.35 e^{-03}$ & $(d)$ \\
$s_{m 2}$ & Background mdm2 synthesis coefficient & 0 & $(e)$ \\
$s_{m 3}$ & mdm2 synthesis coefficient & 2 & $(b)$ \\
$s_{p}$ & p53 synthesis rate & 0.5 & $(e)$ \\
$Z$ & DNA damage & 1 & \\
\hline
\end{tabular}

[3] D. Bennett. Applications of Delay Differential Equations in Physiology and Epidemiology. $\mathrm{PhD}$ Thesis, University of Surrey, 2005.

[4] S. Bernard, B. Cajavec, L. Pujo-Menjouet, M.C. Mackey, H. Herzel. Modelling transcriptional feedback loops: the role of Gro/TLE1 in Hes1 oscillations. Phil. Trans. R. Soc. A, 364 (2006), 1155-1170.

[5] A. Ciliberto, B. Novak, J.J. Tyson. Steady states and oscillations in the p53/mdm2 network. Cell Cycle, 4 (2005), 488-493.

[6] M.L. Dequeant, E. Glynn, K. Gaudenz, M. Wahl, J. Chen, A. Mushegian, O. Pourquie. A complex oscillating network of signaling genes underlies the mouse segmentation clock. Science, 314 (2006), 1595-1598.

[7] B. Ermentrout. Simulating, Analyzing, and Animating Dynamical Systems: A Guide to XPPAUT for Researchers and Students. Society for Industrial and Applied Mathematics, Philadelphia, 2002.

[8] C.P. Fall, E.S. Marland, J.M. Wagner, J.J. Tyson. Interdisciplinary Applied Mathematics, Mathematical Biology: Computational Cell Biology. Springer-Verlag, New York, 2002. 
[9] R.S. Foo, Y.J. Nam, M.J. Ostreicher, M.D. Metzl, R.S. Whelan, C.F. Peng, A.W. Ashton, W. Fu, K. Mani, S.F. Chin, E. Provenzano, I. Ellis, N. Figg, S. Pinder, M.R. Bennett, C. Caldas, R.N. Kitsis. Regulation of p53 tetramerization and nuclear export by ARC. Proc. Natl. Acad. Sci. USA, 104 (2007), 20826-20831.

[10] S.J. Gallagher, R.F. Kefford, H. Rizos. The ARF tumour suppressor. Intl. J. Biochem. Cell Biol., 38 (2006), 1637-1641.

[11] Y. Haupt, R. Maya, A. Kazaz, M. Oren. Mdm2 promotes the rapid degradation of p53. Nature, 387 (1997), 296-299.

[12] H. Hirata, S. Yoshiura, T. Ohtsuka, Y. Bessho, T. Harada, K. Yoshikawa, R. Kageyama R. Oscillatory expression of the bHLH factor Hes1 regulated by a negative feedback loop. Science, 298 (2002), 840-843.

[13] T.R. Hupp, A. Sparks, D.P. Lane. Small peptides activate the latent sequence-specific DNA binding function of p53. Cell, 83 (1995), 237-245.

[14] B.N. Kholodenko. Cell signalling dynamics in time and space. Nat. Rev. Mol. Cell Biol., 7 (2006), 165-176.

[15] S. Krishna, M.H. Jensen, K. Sneppen. Minimal model of spiky oscillations in NF- $\kappa B$. Proc. Natl. Acad. Sci. USA, 103 (2006), 10840-10845.

[16] A. Kusumi, S. Yasushi, Y. Mutsuya. Confined lateral diffusion of membrane receptors as studied by single particle tracking (nanovid microscopy). Effects of calcium-induced differentiation in cultured epithelial cells. Biophys. J., 65 (1993), 2021-2040.

[17] G. Lahav, N. Rosenfield, A. Sigal, N. Geva-Zatorsky, A.J. Levine, M.B. Elowitz, U. Alon. Dynamics of the p53-mdm2 feedback loop in individual cells. Nat. Gen., 36 (2004), 147-150.

[18] R. Lev Bar-Or, R. Maya, L.A. Segel, U. Alon, A.J. Levine, M. Oren. Generation of oscillations by the p53-mdm2 feedback loop: a theoretical and experimental study. Proc. Natl. Acad. Sci. USA, 97 (2000), 11250-11255.

[19] J. Lewis. Autoinhibition with transcriptional delay: a simple mechanism for the Zebrafish somitogenesis oscillator. Curr. Biol., 13 (2003), 1398-1408.

[20] H. Lodish, A. Berk, P. Matsudaira, C.A. Kaiser, M. Krieger, M.P. Scott, S.L. Zipursky, J. Darnell. Molecular Cell Biology. W.F. Freeman and Company, New York, 2003.

[21] J. Luo, M. Li, Y. Tang, M. Laszkowska, R.G. Roeder, W. Gu. Acetylation of p53 augments its site-specific DNA binding both in vitro and in vivo. Proc. Natl. Acad. Sci. USA, 101 (2004), 2259-2264. 
[22] L. Ma, J. Wagner, J.J. Rice, W. Hu, A.J. Levine, G.A. Stolovitzky. A plausible model for the digital response of p53 to DNA damage. Proc. Natl. Acad. Sci. USA, 102 (2005), 1426614271.

[23] S.M. Mendrysa, M.E. Perry. The p53 tumor suppressor protein does not regulate expression of is own inhibitor, MDM2, except under conditions of stress. Mol. Cell Biol., 20 (2000), 2023-2030.

[24] J. Meyers, J. Craig, D.J. Odde. Potential for control of signaling pathways via cell size and shape. Curr. Biol., 16 (2006), 1685-1693.

[25] G.I. Mihalas, Z. Simon, G. Balea, E. Popa. Possible oscillatory behaviour in p53-mdm2 interaction computer simulation. J. Biol. Syst., 8 (2000), 21-29.

[26] N.A.M. Monk. Oscillatory expression of Hes1, p53, and NF-kappaB driven by transcriptional time delays. Curr. Biol., 13 (2003), 1409-1413.

[27] C.W. Mullineaux, A. Nenniger, N. Ray, C. Robinson. Diffusion of green fluorescent protein in three cell environments in Escherichia coli. J. Bacteriol., 188 (2006), 3442-3448.

[28] D.E. Nelson, A.E. Ihekwaba, M. Elliott, J.R. Johnson, C.A. Gibney, B.E. Foreman, G. Nelson, V. See, C.A. Horton, D.G. Spiler, S.W. Edwards, H.P. McDowell, J.F. Unitt, E. Sullivan, R. Grimley, N. Benson, D. Broomhead, D.B. Kell, M.R. White. Oscillations in NF- $\kappa B$ signaling control de dynamics of gene expression. Science, 306 (2004), 704-708.

[29] S.R. Neves, P. Tsokas, A. Sarkar, E.A. Grace, P. Rangamani, S.M. Taubenfeld, C.M. Alberini, J.C. Schaff, R.D. Blitzer, I.I. Moraru, R. Iyengar. Cell shape and negative links in regulatory motifs together control spatial information flow in signaling networks. Cell, 133 (2008), 666680 .

[30] L. Nie, M. Sasaki, C.G. Maki C.G. Regulation of p53 nuclear export through sequential changes in conformation and ubiquitination. J. Biol. Chem., 282 (2007), 14616-14625.

[31] B.A. Ogunnaike. Elucidating the digital control mechanism for DNA damage repair with the p53-mdm2 system: single cell data analysis and ensemble modelling. J. R. Soc. Interface, 3 (2006), 175-184.

[32] J. Owen. Topological proteomics: a new approach to drug discovery. Drug Discovery Today, 6 (2001), 1081-1082.

[33] I.G. Pearce, M.A.J. Chaplain, P.G. Schoeld, A.R.A. Anderson, S.F. Hubbard. Modelling the spatio-temporal dynamics of multi-species host-parasitoid interactions: heterogeneous patterns and ecological implications. J. Theor. Biol., 241 (2006), 876-886.

[34] S. Pigolotti, S. Krishna, M.H. Jensen. Oscillation patterns in negative feedback loops. Proc. Natl. Acad. Sci. USA, 104 (2007), 6533-6537. 
[35] W. Schubert. Cytomics in characterizing toponomes: towards the biological code of the cell. Cytometry, 69A (2006), 209-211.

[36] J.A. Sherratt, M.A. Lewis, A.C. Fowler. Ecological chaos in the wake of invasion. Proc. Natl. Acad. Sci. USA, 92 (1995), 2524-2528.

[37] J.A. Sherratt, B.T. Eagen, M.A. Lewis. Oscillations and chaos behind predatorprey invasion: mathematical artifact or ecological reality? Philos. Trans. R. Soc. London B, 52 (1997), 79-92.

[38] J.A. Sherratt. Periodic travelling waves in cyclic predatorprey systems, Ecol. Lett., 352 (2001), 21-38.

[39] Shieh S.Y., Ikeda M., Taya Y., Prives C. DNA damage-induced phosphorylation of p53 alleviates inhibition by MDM2. Cell, 91 (1997), 325-334.

[40] Srividya, J., Gopinathan, M.S., Schnells, S. The effects of time delays in a phosphorylationdephosphorylation pathway, Biophys. Chem., 125 (2007), 286-297.

[41] Y. Tang, W. Zhao, Y. Chen, Y. Zhao, W. Gu. Acetylation is indispensable for p53 activation. Cell, 133 (2008), 612-626.

[42] G. Tiana, M.H. Jensen, K. Sneppen. Time delay as a key to apoptosis induction in the p53 network, Eur. Phys. J. B, 29 (2002), 135-140.

[43] G. Tiana, S. Krishna, S. Pigolotti, M.H. Jensen, K. Sneppen. Oscillations and temporal signalling in cells. Phys. Biol., 4 (2007), R1-R17.

[44] K.H. Vousden, D.P. Lane. p53 in health and disease. Nat. Mol. Cell Biol., 8 (2007), 275-283.

[45] J. Wagner, L. Ma, J.J. Rice, W. Hu, A.J. Levine, G.A. Stolovitzky. p53-mdm2 loop controlled by a balance of its feedback strength and effective dampening using ATM and delayed feedback. I.E.E. Proc. Syst. Biol., 152 (2005), 109-118.

[46] C. Wawra, M. Kuhl, H.A. Kestler. Extended analyses of the Wnt/ $\beta$-catenin pathway: robustness and oscillatory behaviour. FEBS Lett., 581 (2007), 4043-4048.

[47] O. Wolkenhauer, M. Ullah, P. Wellstead, K.H. Cho. The dynamic systems approach to control and regulation of intracellular networks. FEBS Lett., 579 (2005), 1846-1853.

[48] A. Zauberman, D. Flusberg, Y. Haupt, Y. Barak, M. Oren. A functional p53-response intronic promoter is contained within the human mdm2 gene. Nucleic Acids Res., 23 (1995), 25842592.

[49] W.B.J. Zimmerman. Multiphysics Modeling With Finite Element Methods, Series on Stability, Vibration and Control of Systems, Series A - Vol.18. World Scientific Publishing Company, London, 2006. 